\title{
Rapid diagnosis of neonatal sepsis in pediatric population in tertiary care hospital, Durg
}

\author{
Shiv Kumar Chandraker ${ }^{1}$, Smita Bawankar ${ }^{2}$, Animesh Panda ${ }^{3}$, Dhruba Hari Chandi ${ }^{4}$ \\ ${ }^{1}$ Associate Professor of Pathology, CCM Medical College \& Hospital, Durg, ${ }^{2}$ Assistant Professor of Microbiology, CCM Medical College \& \\ Hospital, Durg, ${ }^{3}$ Assistant Professor of Microbiology, CCM Medical College \& Hospital,Durg, ${ }^{4}$ Demonstrator of Microbiology, CCM Medical \\ College \& Hospital, Durg
}

Background: In under developing country Septicemia in infants to be of common occurrence with high morbidity \& mortality. Aims \& Objectives: Detection of rapid method for diagnosis of sepsis in paediatric age groups. Materials \& Methods: Clinically suspected 369 cases of Bacteraemia in neonates, infants \& children admitted as inpatients at CCM Medical College \& Hospital, Durg \& 45 healthy children as control were included in the present study. The cases were investigated by blood culture \& 5 rapid tests Viz total leucocyte count (TLC), immature to total neutrophil (I: T) ratio, C - reactive protein (CRP), ESR \& Grams smears of Buffy coat for organisms. Results: Blood cultures were positive in 171 (46.34\%) of 369 cases and negative organisms was $55.55 \%$ as against $44.44 \%$ of Gram positive bacteria. The most common isolates were Staph epidermides $(24.56 \%)$ and Staph aureus $(16.37 \%)$ with overall staphylococcal prevalence of $40.93 \%$ followed by gram negative bacteria, S.typhi $(14.61 \%)$ E.coli $11.11 \%$ \& Ps.auroginosa $10.52 \%$. Conclusion: The rapid tests were evaluated in blood culture positive \& negative cases CRP yielded maximum sensitivity of $70.76 \%$, Specificity of $76.26 \%$ \& positive predictive accuracy of $72.02 \%$. Combination of 2 tests did not reveal any significant advantage over single CRP test.

Key words: Bacteraemia, Blood cultures, Rapid diagnostic test \& $\mathrm{CCMMCH}$, Durg

\section{INTRODUCTION}

In under developed and developing countries like India infectious diseases in infants and children are often continued to be of common occurrence with high morbidity and mortality. ${ }^{1}$ Clinically bacteraemia is spread by transplacentally after maternal infection and invasion of the bloodstream is the usual route by which the foetus becomes infected, often difficult to diagnose due to presenting non specific clinical features with no noticeable focus of infection. ${ }^{2}$ Since blood cultures are difficult in neonates, infants and very young children and usually require 2-3 days for diagnosis, some rapid tests have been implemented for early diagnosis of bacteraemia ${ }^{3}$ to facilitate prompt treatment.

\section{MATERIALS AND METHODS}

The clinical material on which this study is based, were obtained from CCM Medical College \& Hospital which comprised of 369 clinically suspected cases of bacteriaemia in children with 45 healthy children serving as controls.

Blood samples were collected aseptically for different tests depending upon the age of the child. About $3-5 \mathrm{ml}$ blood was drawn from children of age 6 months and above. One $\mathrm{ml}$ of blood was put into a bottle containing $2 \mathrm{mg} / \mathrm{ml}$ EDTA as anticoagulant for TLC, I: T neutrophil ratio and buffy coat. One $\mathrm{ml}$ was allowed to clot in a sterile bijou bottle for CRP and $0.5 \mathrm{ml}$ was collected in a sterile small test tube with $0.2 \mathrm{ml} \mathrm{3.8 \%} \mathrm{citrate} \mathrm{solution} \mathrm{for} \mathrm{ESR.} \mathrm{Remaining}$ $2.3 \mathrm{ml}$ blood was inoculated into $20 \mathrm{ml}$ Trypticase soya broth (TSB) containing 0.05\% liquid in McCartney bottle. In neonates and infants finger prick blood was used for TLC, I:T ratio \& at least $1 \mathrm{ml}$ blood was collected and clotted in a bottle for CRP and clot culture in $10 \mathrm{ml}$ TSB.

The samples were immediately processed in the laboratory. Blood cultures \& clot cultures were incubated at $37^{\circ} \mathrm{C}$ for 
10 days with subcultures at 3 days intervals on blood agar \& MacConkey agar. The bacterial isolates were identified by biochemical reactions \& special tests. ${ }^{4}$ TLC was made using improved Neubaur counting chamber \& WBC pipette and I:T ratio in Leishman's blood smears by making differential count of 100 successive neutrophils to determine nuclear indices according number of lobes of nuclei. Gram's smears of buffy coat obtained by centrifugation of EDTA blood in Wintrobe tube at $2500 \mathrm{rpm}$ were examined microscopically for organisms. CRP estimation was done by latex agglutination using reagents obtained from Tulip Diagnostics limited.

The cut off values for positive tests were TLC less than 5000 and more than $20000 / \mathrm{cmm}$; I: T neutrophil ratio 0.2 and above; ESR more than $10 \mathrm{~mm}$ of first hour; CRP more than $6 \mathrm{mg} / \mathrm{ml}$. The results of all the rapid tests were analyzed singly or in combination of 2 to assess their sensitivity, specificity and positive predictive accuracy.

\section{RESULTS}

Out of 369 cultures which includes blood and clot cultures, $171(46.34 \%)$ yielded growth and all blood cultures were negative in controls (Table 1). All positive blood cultures revealed only monobacterial isolates. Out of 171 cultures, positive $95(55.55 \%)$ showed growth of Gram negative bacteria and $76(44.44 \%)$ yielded Gram positive organisms.

It is also evident from Table 2 that the most common isolates were Staphylococcus epidermidies $(24.56 \%)$ and Staphylococcus aureus (16.37\%) giving overall Staphylococcal prevalence of $40.93 \%$ followed by Gram negative bacteria which includes S.Typhi (14.61\%), E.coli $(11.11 \%)$ and Ps.auroginosa $10.52 \%$.

Table 3 shows comparison between culture positive \& culture negative isolates which are collected from Blood culture out of 369 clinical patients.

Table 4 shows the result of rapid diagnostic tests in 171 positive culture and 198 negative cultures cases. Gram smears of buffy coat were negative for organisms in both positive and negative cultures cases. But out of 171 blood culture positive cases, abnormal values of TLC in $98(57.30 \%)$,I: T ratio in 89 (52.04\%), ESR in 91 (53.21\%) and CRP in $121(70.76 \%)$ were observed. On the other hand, out of 198 negative culture cases, abnormal values of TLC in $86(43.43 \%)$, I: T ratio 104 (52.52\%), ESR $56(28.28 \%)$ and in $151(76.26 \%)$ were noticed.

Table 5 shows the results of sensitivity, specificity and positive predictive accuracy of 4 rapid diagnostic tests either singly or in combination of 2 tests were recorded

\begin{tabular}{lcc}
\multicolumn{4}{l}{ Table 1: Number and percentage of different group } \\
\multicolumn{3}{l}{ organisms out of $\mathbf{1 7 1}$ positive blood cultures } \\
\hline Organisms & Number & Percentage \\
\hline Gram positive isolates & 76 & 44.44 \\
Gram negative isolates & 95 & 55.55 \\
Total & 171 & 100 \\
\hline
\end{tabular}

\begin{tabular}{lcc}
\multicolumn{3}{l}{$\begin{array}{l}\text { Table 2: Number and percentage of different types } \\
\text { of organisms out of } \mathbf{1 7 1}\end{array}$} \\
positive blood cultures \\
\hline Organisms & Number & Percentage \\
\hline Staphylococcus epidermidies & 42 & 24.56 \\
Staphylococcus aureus & 28 & 16.37 \\
Candida albicans & 06 & 03.50 \\
Salmonella typhi & 25 & 14.61 \\
Escherichia coli & 19 & 11.11 \\
Pseudomonas aeruginosa & 18 & 10.52 \\
Proteus mirabilis & 14 & 08.18 \\
Klebsiella aerogenes & 12 & 07.01 \\
Citrobacter freundii & 07 & 04.09 \\
Total & 171 & 100 \\
\hline
\end{tabular}

\begin{tabular}{lcc}
$\begin{array}{l}\text { Table 3: Number and percentage of culture } \\
\text { positive and negative organisms out of } \mathbf{3 6 9} \\
\text { clinically suspected inpatients }\end{array}$ \\
\hline Blood culture & Number & Percentage \\
\hline Culture positive & 171 & 46.34 \\
Culture negative & 198 & 53.65 \\
Total & 369 & 100 \\
\hline
\end{tabular}

in Table 3. It is evident that CRP revealed sensitivity of $70.76 \%$, specificity of $76.26 \%$ and positive predictive accuracy of $72.02 \%$, when compared with other tests either alone or in combination of 2 tests.

\section{DISCUSSION}

Bacteraemia in paediatric cases seen in hospital and clinics is of frequent occurrence with serious sequelae. Salient clinical features and some rapid laboratory tests often help to make early diagnosis. ${ }^{5}$

The overall smear rate of blood cultures in the present study is $46.34 \%$. The reported positive blood cultures by different Indian authors were $50.0 \%,{ }^{6} 60.0 \%,{ }^{7} 59.9 \%{ }^{8}$ and $32.0 \%$.

Our study revealed prevalence of Gram negative bacteria as high as $55.55 \%$ when compared to prevalence of Gram positive organisms $44.44 \%$.Further more, we observed Staphylococcal predominance of $40.93 \%$ with prevalence of Staphylococcus epidermidies and Staphylococcus aureus being $24.56 \%$ and $16.37 \%$ followed by S.typhi $(14.61 \%)$, E.coli (11.11) and Ps. aeruginosa (10.52\%). Moreover our study reported $4.09 \%$ isolation rate of Citrobacter freundii. 


\begin{tabular}{|c|c|c|c|c|c|c|c|c|}
\hline Test & Positive (A) & $(\%)$ & Negative (C) & $(\%)$ & Negative (B) & $(\%)$ & Positive (D) & $(\%)$ \\
\hline TLC & 98 & 57.30 & 73 & 42.69 & 112 & 56.56 & 86 & 43.43 \\
\hline I:T neutrophil ratio & 89 & 52.04 & 82 & 47.95 & 94 & 47.47 & 104 & 52.52 \\
\hline ESR & 91 & 53.21 & 80 & 46.78 & 142 & 71.71 & 56 & 28.28 \\
\hline CRP & 121 & 70.76 & 50 & 29.23 & 47 & 23.23 & 151 & 76.26 \\
\hline Buffy coat smear & 0 & 0 & 0 & 0 & 0 & 0 & 0 & 0 \\
\hline
\end{tabular}

\begin{tabular}{|c|c|c|c|}
\hline Test & $\begin{array}{c}\text { Sensitivity } \\
(A \times 100 / A+C)\end{array}$ & $\begin{array}{c}\text { Specificity } \\
(D \times 100 / B+D)\end{array}$ & $\begin{array}{c}\text { Positive } \\
\text { predictive } \\
\text { accuracy } \\
(\mathrm{A} \times 100 / \mathrm{A}+\mathrm{B})\end{array}$ \\
\hline TLC & 57.30 & 43.43 & 46.66 \\
\hline I:T ratio & 52.05 & 52.52 & 48.64 \\
\hline ESR & 53.22 & 28.28 & 39.05 \\
\hline CRP & 70.76 & 76.26 & 72.02 \\
\hline CRP+TLC & 64.03 & 59.84 & 59.51 \\
\hline $\mathrm{CRP}+\mathrm{I}: \mathrm{T}$ ratio & 61.40 & 64.39 & 59.82 \\
\hline CRP+ESR & 61.99 & 52.01 & 52.86 \\
\hline
\end{tabular}

Out of 5 rapid tests, Gram smears of buffy coat were significantly negative in all cases. CRP showed maximum sensitivity of $70.76 \%$, specificity of $76.26 \%$ and positive predictive accuracy of $72.02 \%$ and these findings are in agreement with the reports of other authors. ${ }^{10,11}$ On the other hand other rapid tests singly or in combinations of 2 did not show any advantage when compared to CRP test alone. Based on our observations, we are of the opinion that blood cultures and a battery of rapid tests could be carried out depending upon the amount of blood drawn from children of different ages groups. And if blood drawn is around $1 \mathrm{ml}$ only CRP test could be preferred since it is a sensitive indicator of bacteraemia in the absence of blood cultures. Clot cultures could be done whenever possible.

\section{CONCLUSION}

Under the observation of neonatal sepsis, out of 5 rapid tests, CRP is the most important rapid test for the diagnosis of neonatal sepsis.

\section{ACKNOWLEDGMENT}

We are extremely thankful to the teaching staff of the Department of pediatrics, CCM Medical College \& Hospital, Durg for their kind permission to carry out this study. Technical help from the Department of Microbiology.

\section{REFERENCES}

1. Goldman DA, Freeman J and Durbin WA. Nosocomial infection and death in a neonatal intensive care unit. J inf Dis 1983; 147:635-639.

2. Siegel JD and McCraken GH. Sepsis neonatorum N Eng J Med 1981; 304:642-645.

3. Kite M, Miller MR, Gorham P and Congclon P. Comparision of files test in diagnosis of neonatal bacteaemia. Arch Dis child 1988; 63: 639-643.

4. Collee JG and Miles RS. Mackie and McCartney. Practical medical Microbiology, test for identification of bacteria $13^{\text {th }}$ ed (Churchill lifingston 1989), pp 141-165.

5. Mc Lellan D and Glebink GS. Perspectives of occult bacteraemia in children. J Paediatr 1986; 109:1-8.

6. Namdev UK, Singh HP, Rajput VJ and Kushwaha JS. Haematological indices for early diagnosis of neonatal septicaemia. Ind J Paediatr 1985; 22: 287-292.

7. Mehrotra N, Kumar A, Chansoria M and Kaul KK. Neonatal Sepsis, Correlation of maternal \& neonatal factors to positive bacterial cultures.

8. Khatua SO, Das AK, Chatterijee BD, Khatua S, Ghose B and Saha A. Neonatal Sepsis. Ind J Paediatr 1986; 53: 509-514.

9. Bhakoo ON. Neonatal bacterial infections in Chandigarh; a decade of experience. Ind J Paediatr 1980; 47: 419-424.

10. Anuradha DE, Kari S, Gogate A and Raghavan K. C-reactive protein and buffy coat smear in early diagnosis of neonatal Septicaemia. Ind J Pathal Microbiol 1998; 41: 23-26.

11. Anita Sharma CV, Kutty K, Sabharwal U, Rathee $S$ and Mohan $\mathrm{H}$. Evalution of Sepsis serene for diagnosis of neonatal Septicaemia. Ind J Paediatr 1993; 60: 559-563. 